\title{
Initial port integration concept for EC and NB systems in EU DEMO tokamak
}

\author{
Thomas Franke ${ }^{1,2}$, Piero Agostinetti ${ }^{3}$, Christian Bachmann $^{1}$, Alessandro Bruschi ${ }^{4}$, Matthew Carr ${ }^{5}$, \\ Fabio Cismondi ${ }^{1}$, Aljaz Cufar ${ }^{6}$, H.P.L. de Esch ${ }^{7}$, Gianfranco Federici ${ }^{1}$, Niek den Harder ${ }^{2}$, Saul \\ Garavaglia $^{4}$, Giovanni Grossetti ${ }^{8}$, Gustavo Granucci ${ }^{4}$, Alex Meakinss, Alessandro Moro ${ }^{4}$, Rocco \\ Mozzillo $^{9}$, Emanuele Sartori ${ }^{3}$, Mattia Siccinio ${ }^{1}$, Piergiorgio Sonato ${ }^{3}$, Dirk Strauss ${ }^{8}$, Minh Quang \\ $\operatorname{Tran}^{10}$, Alex Valentine ${ }^{5}$, Shanliang Zheng ${ }^{5}$ \\ ${ }^{I}$ EUROfusion Power Plant Physics and Technology (PPPT) department, Garching, Germany \\ ${ }^{2}$ Max-Planck-Institut für Plasmaphysik, Garching, Germany \\ ${ }^{3}$ Consorzio RFX (CNR, ENEA, INFN, Università di Padova), Padova, Italy \\ ${ }^{4}$ IFP-CNR, Istituto di Fisica del Plasma "P. Caldirola", Milano, Italy \\ ${ }^{5}$ Culham Centre for Fusion Energy, Abingdon, OX14 3DB, Oxfordshire, U.K. \\ ${ }^{6}$ Reactor Physics Department, Jožef Stefan Institute, Jamova cesta 39, SI-1000, Ljubljana, Slovenia \\ ${ }^{7}$ CEA-IRFM, F-13108 Saint-Paul-Lez-Durance, France \\ ${ }^{8}$ Karlsruhe Institute of Technology, P.O. Box 3640, D-76021 Karlsruhe, Germany \\ ${ }^{9}$ CREATE, University of Naples Federico II, DII, P.le Tecchio 80, 80125, Naples, Italy \\ ${ }^{10}$ Swiss Plasma Center EPFL, CH-1015 Lausanne, Switzerland
}

\begin{abstract}
The integration of the heating and current drive (HCD) systems in the EU DEMO tokamak must address a number of issues, namely space constraints in the tokamak building, remote handling requirements, breeding blanket penetration, neutron and photon radiation shielding, compliance of penetrations of the primary vacuum with safety and vacuum criteria, and a large number of loading conditions, in particular heat, electromagnetic (EM), and pressure loads in normal and off-normal conditions. A number of pre-conceptual design options for the vacuum vessel (VV) port and the port-plug are under assessment and need to be verified against all requirements and related criteria. The identification of the functional (or physics) requirements of the HCD systems remains an on-going process during the pre-conceptual design phase, hence some initial assumptions had to be made as a basis for development of the design of the VV ports and the HCD port plugs.

The paper will provide an overview of present margins in the functional/physics requirements and the rationale behind the assumptions made in order to facilitate development of the pre-conceptual design options. Furthermore it will introduce the initial design concepts of the electron cyclotron (EC) Launchers and the neutral beam (NB) injectors integrated in equatorial ports. The NB duct design in DEMO and related issues such as transmission and re-ionization losses will be also addressed.
\end{abstract}

Keywords: Plasma heating and current drive, Electron cyclotron, Neutral beam injection, Port integration

\section{Introduction}

The development of a demonstration fusion power plant is one of the missions of the European fusion strategy. The major design parameter of the present EU DEMO baseline as given in [1][2], is that its plasma requires auxiliary heating and current drive (HCD) power. The HCD mix will be decided at a later stage of the project. Presently it is assumed that the power could be delivered by one or more of the systems under study, electron cyclotron $(\mathrm{EC})$, neutral beam $(\mathrm{NB})$ or ion cyclotron (IC). Each system has a development target of $50 \mathrm{MW}$. A power requirement of $>100 \mathrm{MW}$ of launched power which could be integrated into DEMO is assumed (cf. chapter 2). The review panel (RP) of the work package HCD recommended changing the strategy for the IC integration in order to decouple the two complex systems breeding blanket (BB) and HCD and improve the physics performance. This RP recommendation is presently under study. Therefore the new IC integration cannot yet be presented in this paper.

\section{Physics requirements}

The latest information about the DEMO plasma scenario is given in [3]. For the EU DEMO baseline (2017) the previous definition for HCD power was used, i.e. $50 \mathrm{MW}$ for the plasma flat top (FT), as input to the system code (PROCESS [4]) that determines the DEMO build-up. Transient phases (breakdown, ramp-up, rampdown and dwell) in view of HCD are not covered by this code. Studies are done to estimate the transient power requirements, e.g. [5][6]. Table 1 summarizes the auxiliary power for the main plasma phases of DEMO. The power for the EC assisted breakdown is not listed as it is only a few MWs and still under investigation [6]. Also the HCD dwell power is not listed and assumed to be zero MW.

For the ramp-up phase the L-H threshold power $\mathrm{P}_{\mathrm{LH}}$ is $120 \mathrm{MW} \pm 20 \mathrm{MW}$ according to the Martin scaling law [7]. Although studies were conducted to enter $\mathrm{H}$-mode at lower density and somewhat lower power, the present 
assumption is to use this $\mathrm{L}-\mathrm{H}$ threshold value as the functional requirement. In presently considered scenarios there is a necessity to prevent strong magnetohydrodynamic (MHD) activities by applying $30 \mathrm{MW}$ of EC power for sawtooth (ST) control at somewhat outside of $\mathrm{q} \approx 1$ which will be alternating with the pre-emptive neoclassical tearing mode (NTM) [8] control at $q=2 / 1$ and $q=3 / 2$ with $15 \mathrm{MW}$ for each of the modes [6]. For the latter value error bars might be in the range of several MWs, and are strongly connected to the detection and actuator response times and the time evolution of the magnetic island. It should be mentioned that for the present DEMO baseline, no bulk current drive is foreseen, whereas for alternative scenarios like Flexi-DEMO [9] it is $>100 \mathrm{MW}$ of launched power.

Table 1. Power requirements EU DEMO launched to the plasma by HCD systems, - underlined is continuous power, - italic is power included by dominating power and will relax the before mentioned to the same amount, - bold numbers are dominating power requirements

\begin{tabular}{|c|c|c|c|}
\hline $\begin{array}{l}\text { Function vs. } \\
\text { plasma phases, } \\
\text { power in }[M W]\end{array}$ & $\begin{array}{l}\text { Ramp- } \\
\text { up }\end{array}$ & $\begin{array}{l}\text { Flat- } \\
\text { top }\end{array}$ & $\begin{array}{l}\text { Ramp- } \\
\text { down }\end{array}$ \\
\hline$L-H$ heating & $120 \pm 20$ & & \\
\hline$S T, N T M^{2}$ & $30 / 15 *$ & $30 / 15 *$ & 30/15* \\
\hline Burn control ${ }^{-}$ & 50 & 50 & 50 \\
\hline $\begin{array}{l}\text { Radiative in- } \\
\text { stability control }\end{array}$ & & 40 & \\
\hline $\begin{array}{l}\text { Ramp-down } \\
\text { control }^{4}\end{array}$ & & & $100 \pm 30$ \\
\hline Max. intermittent & $120 \pm 20$ & 50 & $130 \pm 30$ \\
\hline
\end{tabular}

${ }^{1}$ core heating, ${ }^{2}$ local ECCD, ${ }^{3}$ off-axis heating to avoid H-L transition, ${ }^{4}$ heating at non-specified location, work is in progress, * in case of contemporary mode control $(2 / 1)$ and $(3 / 2)$ the NTM power duplicates to $30 \mathrm{MW}$

\section{Port integration}

The integration for the two systems EC and NB is an ongoing process [10]-[12] and is supported by functional analyses. For further details on the methodology for the HCD interface and requirements analysis, a systems architecture model was established [13]. The conceptual design of the systems including sub-systems are described elsewhere cf. for EC [6][10][12], for NB [14][15]. Fig. 1 shows one of several configurations currently under study, at time of writing the IC was integrated at upper position in the $\mathrm{BB}$, this will change because of RP recommendations as mentioned under chapter 1. Afterwards an IC midplane installation is foreseen, requiring re-arrangements of ports. Also the number of required ramp-up limiters shall be fixed at the end of the present pre-conceptual design stage of DEMO.

Amongst the decisions yet to be made are the number of ramp-up limiters (LIM), the number of diagnostic ports (DC), and depending on the choice of HCD systems, the number and type of EC, NB, and IC ports. The use of a multi-purpose deployer (MPD) in the limiter ports during removal of the limiters as well as the implementation of the disruption mitigation system (DMS) and thermography devices in the limiter port plugs is being studied. The outcome may vary the allocation of the equatorial ports in the future.

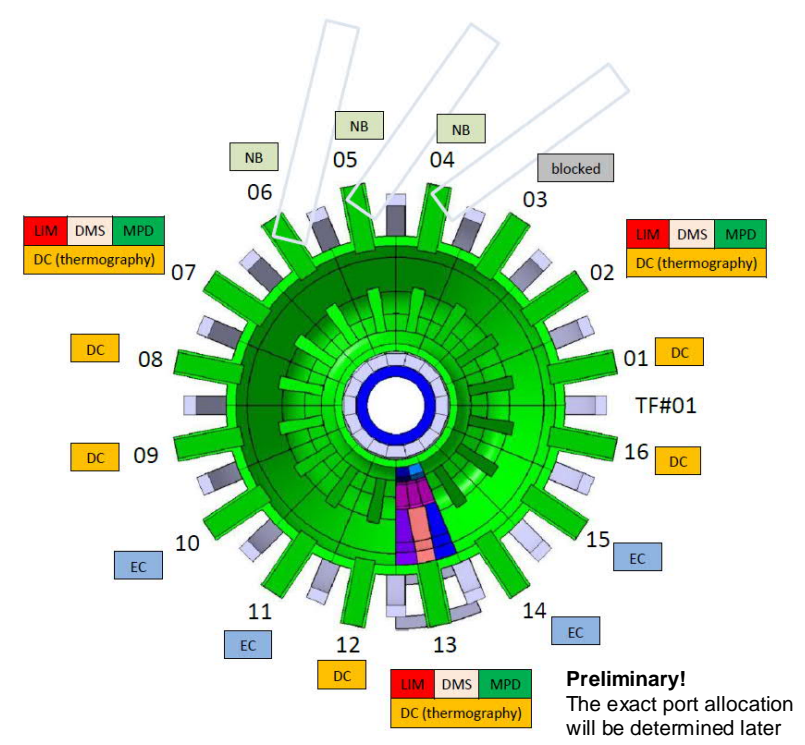

Fig. 1. Draft allocation of the equatorial ports assuming 3 ramp- up limiters

\subsection{Neutral beam duct design}

With regards to remote maintenance (RM) and under optimization of former NB concept designs [14][15], the NB duct was re-designed to have a funnel-type shape (Fig. 2) instead of parallel side walls to adapt for the modular sources concept. Vacuum pumping of the NB duct may not be necessary and is under study. The inside of the duct is composed of a duct liner with high-heatflux components supported by the duct liner neutron shield. The beam focal point has been moved from the $\mathrm{BB}$ center to the first wall (FW) maintaining the same transmission efficiency as before. As the beamlets were focused previously in the BB center and they are now focused at the FW, the opening in the FW could be reduced from $0.7 \times 0.7 \mathrm{~m}^{2}$ [16] to $0.5 \times 0.5 \mathrm{~m}^{2}$. This reduces the neutron streaming through the NB port.

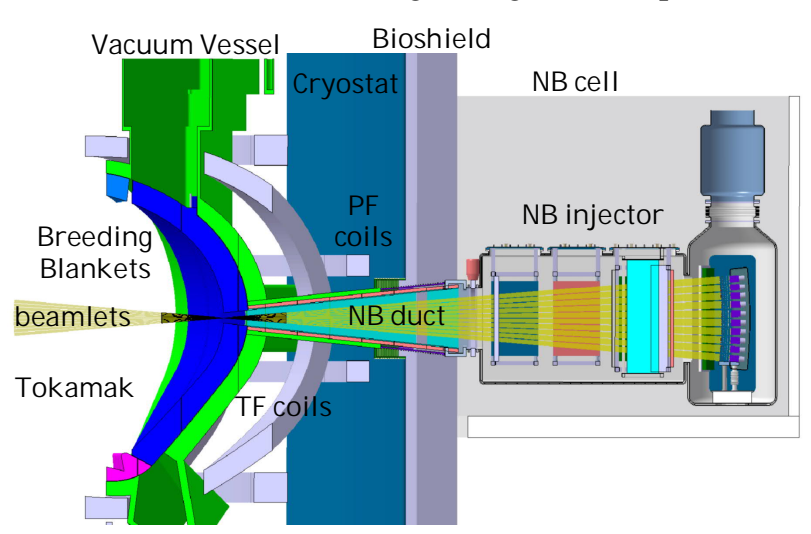

Fig. 2. Side view of the DEMO NB CAD design.

The losses (re-ionization, transmission, neutron heating and plasma radiation wall loads) define the design of the cooling and based on neutronic results consequently the material selection in the duct. The NB duct liner is considered to be a lifetime component and a replacement is therefore not needed, which makes a robust design and the proper choice of material crucial. 


\subsubsection{NB duct losses due to re-ionization}

The re-ionization losses were studied and results are provided in [17]. The power in the re-ionised particles

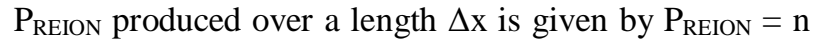
$\sigma \Delta \mathrm{x} \mathrm{P}_{\mathrm{NBI}}$ with $\mathrm{n}$ being the gas density, $\sigma$ the reionisation cross section and $\mathrm{P}_{\mathrm{NBI}}$ the neutral beam power. The re-ionized particles are deflected along the field lines for which 3D electric field maps were produced along the duct. The calculated re-ionization duct losses are rather low with a total of $65 / 72 \mathrm{~kW}$ for the duct right/left side walls. It could be seen that the toroidal fringing field is dominant in the duct region. Therefore the re-ionization losses at the floor/roof side walls are at a very low level with $0 / 2 \mathrm{~kW}$.

\subsubsection{NB transmission losses}

Fig. 3 shows the power densities for core and halo beams in the FW opening (red square), where all the single beamlets pass the focus point. The total injected power to the plasma is $16.8 \mathrm{MW}$, which is the DEMO NB requirement [14]. The extensions of the FW opening for the core beam are sufficient, whereas in view of the halo beam the vertical size is the limiting factor and needs to be slightly increased, taking into account that losing a big fraction of the halo power (which covers only $15 \%$ of the total beam power) is unavoidable, otherwise the opening would be too big for any practical integration purposes and neutronic considerations as well.

Furthermore it turned out during the transmission calculations that the NB components inside the NB injector are not perfectly aligned in order to achieve a required high beam line/duct transmission efficiency of $92 \%$, given as DEMO design target. The NB injector is not part of the work done here and will be sorted out by re-iteration with the injector design team.
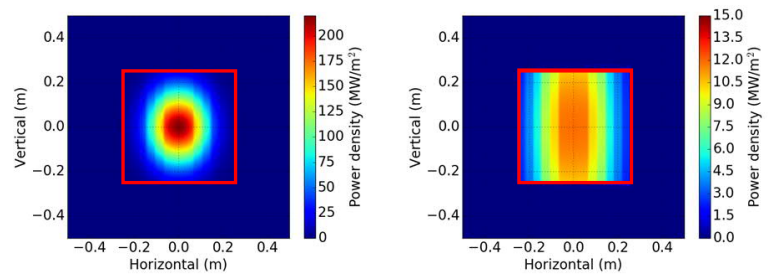

Fig. 3. Beam core (left) / halo (right) for an assumed $7 \mathrm{mrad} /$ $30 \mathrm{mrad}$ beam through the opening in the BB FW (red square).

Fig. 4 left shows the relative difference in beam transmission from the modular sources with all injector components and the duct (red curve) and for the duct only (blue curve). Fig. 4 right shows the power density profiles for single beamlets at the FW opening. At larger divergences the beamlets have a considerable size and significant losses are unavoidable as mentioned before.
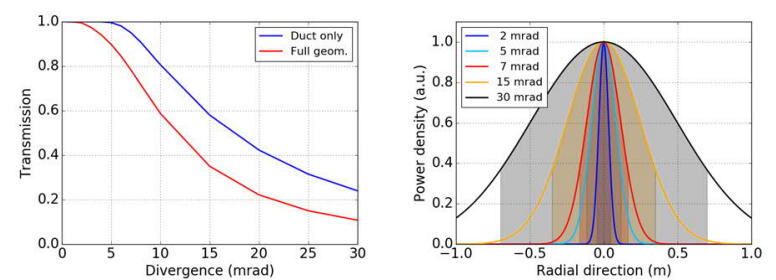

Fig. 4. Left: Transmission curves for full geometry vs. duct only. Right: power spreading for different divergences at the FW opening. The shaded areas indicate where the power density is $1 / \mathrm{e}$ of the on-axis power density.

\subsubsection{NB Neutronic loads}

Coupled neutron and photon transport calculations were conducted [18] based on a former NB port design in MCNP6v1 [19]. The former design is mainly different in the FW opening and duct shape. The calculated neutron flux was coupled with an inventory simulation computed in FISPACT-II [20] using the MCR2S method to output a subsequent shutdown photon source. The results for the former NB design confirmed that beyond the bioshield, inside the NB injector, calculated values of the shut-down dose rate (SDDR) 12 days after shut-down are in the order of $10^{6}-10^{7} \mu \mathrm{Sv} / \mathrm{hr}$ and, outside the NB injector, the calculated dose rate is approximately $10^{5} \mu \mathrm{Sv} / \mathrm{hr}$ [18]. These values are several orders of magnitude above the limit for hands-on operations in DEMO ( 100 $\mu \mathrm{Sv} / \mathrm{hr}[21])$ hence - as in ITER - RM is required even with further shielding optimization.

With the new design, the NB opening was decreased to $0.5 \times 0.5 \mathrm{~m}^{2}$, with translation of the focal point. As a result, it is expected that the updated neutronic values including toroidal field (TF) coil heating and SDDR are decreased and more in line with the design criteria. The limit to the nuclear heating in the TF coil is specified as $5 \times 10^{-5} \mathrm{~W} \mathrm{~cm}^{-3}$ [22]. For the former port dimensions $\left(0.7 \times 0.7 \mathrm{~m}^{2}\right)$, this was exceeded in the outer layers of the winding packs of the TF coils, even after the improvement of shielding structures through extension of the duct liner, see Fig. 5. The analysis of such nuclear quantities with the updated 2017 baseline MCNP model and a revised NBI design is ongoing.

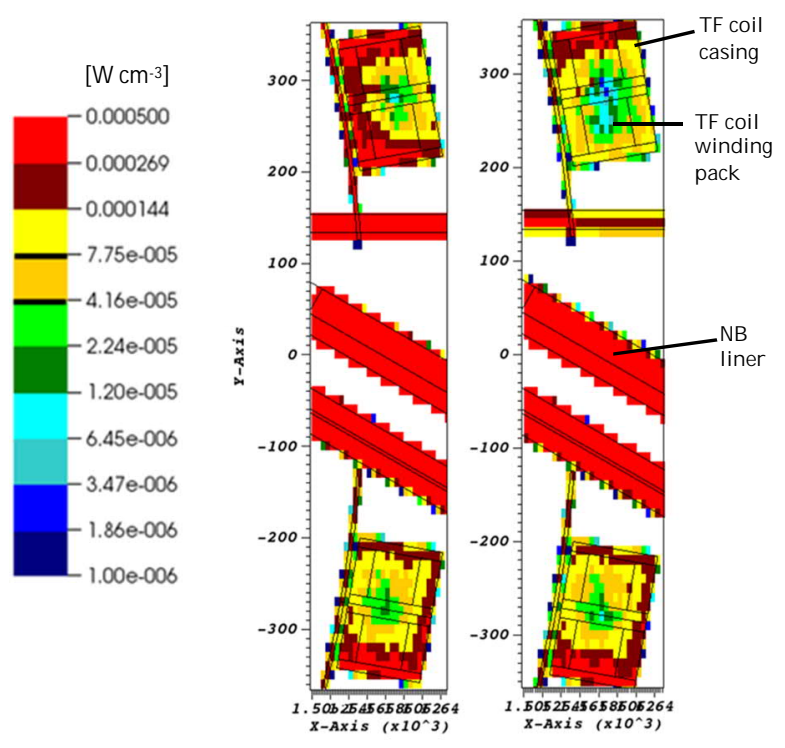


Fig. 5. Comparison of heat density $\left(\mathrm{W} \mathrm{cm}^{-3}\right)$ in the $\mathrm{TF}$ coils without (left) and with (right) improved duct liner shielding for midplane level $(\mathrm{z}=0 \mathrm{~m})$.

\subsubsection{NB Radiation wall loads and shine-through losses}

With a 3D surface meshed model the radiation wall loads and power densities were simulated using raytracing techniques [23]. The values at the front face of the NB opening and at the side walls of the NB aperture of $0.5 \times 0.5 \mathrm{~m}^{2}$ are given in the legend of Fig. 6 .

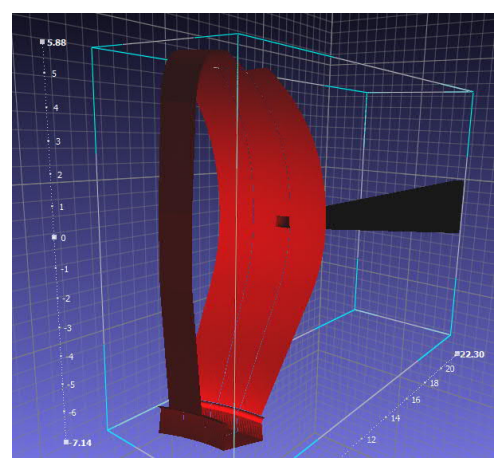

Fig. 6. Simplified NB 3D radiation load mesh model, the strength of the red colouring on the mesh triangles indicates the strength of the power deposited, at the FW light red colour is 350 $\mathrm{kW} / \mathrm{m}^{2}$, at the $\mathrm{NB}$ opening side wall dark red is $215 \mathrm{~kW} / \mathrm{m}^{2}$.

The shine-through losses on the opposite wall from the NB opening were also studied. For the FT phase the shine-through losses were simulated with different injection angles and geometries [24] and are negligible, while during the ramp-up phase significant heat loads would occur on the wall if the beams are switched on before a certain plasma density is reached. During the ramp-up of the plasma current, the shine-through losses were simulated, for results see Table 2 [25].

Table 2. NB shine-through peak power losses (2015 DEMO baseline) during plasma ramp-up up to plasma FT, simulated with Monte Carlo codes [25].

\begin{tabular}{|c|c|c|c|c|c|}
\hline Plasma current & $\begin{array}{c}I_{P} \\
{[M A]}\end{array}$ & 5 & 10 & 15 & $\begin{array}{l}19.6 \\
{[F T]}\end{array}$ \\
\hline $\begin{array}{l}\text { Volume-average } \\
\text { electron density }\end{array}$ & $\begin{array}{c}<n_{e}> \\
{\left[10^{19} \mathrm{~m}^{-3}\right]}\end{array}$ & 0.78 & 1.36 & 2.92 & 6.54 \\
\hline $\begin{array}{l}\text { Shine-through } \\
\text { peak power }\end{array}$ & $\begin{array}{l}P_{\text {shine-through }} \\
{\left[\mathrm{MW} / \mathrm{m}^{2}\right]}\end{array}$ & 1.10 & 0.41 & 0.05 & 0 \\
\hline
\end{tabular}

\subsection{EC launcher design}

The EC launcher design was and will be further studied for different options, which are open ended wave guide (OEWG), remote steering antenna (RSA) and another option shall be introduced, a mid steering antenna (MSA), which in contrast to the ITER front steering antenna (FSA) is protected by the DEMO BB and where the movable parts are inside a port plug which is separated behind the BB. The port plug concept is called blanket separated design (BSD) because it does not penetrate the breeding blanket as opposed to the blanket integrated design (BID), not used in DEMO for EC. Further information on the OEWG and RSA can be found in [10][12]. For the MSA option, the work has started at the end of 2017 and will be continued in the future. Some first results are given below.
A first CAD design of the MSA is shown in Fig. 7. The MSA launcher was mainly designed for heating and instability control as local electron cyclotron current drive (ECCD) is required for this purpose. The main instabilities to be considered for the MSA design are neoclassical tearing modes (NTMs) and sawteeth (ST). The EC MSA launcher is based on four drawers, each fed by eight waveguides, able to handle up to $2 \mathrm{MW}$ of RF power launched each, to guarantee about $50 \mathrm{MW}$ (plus spare) to the plasma. The optical and ray-tracing studies are on-going and therefore the final mirror configuration (number, curvatures, sizes, and distances) and related number of beams per drawer is under examination. The steering ranges shall also cover the transient plasma phases (ramp-up, -down) for NTMs.
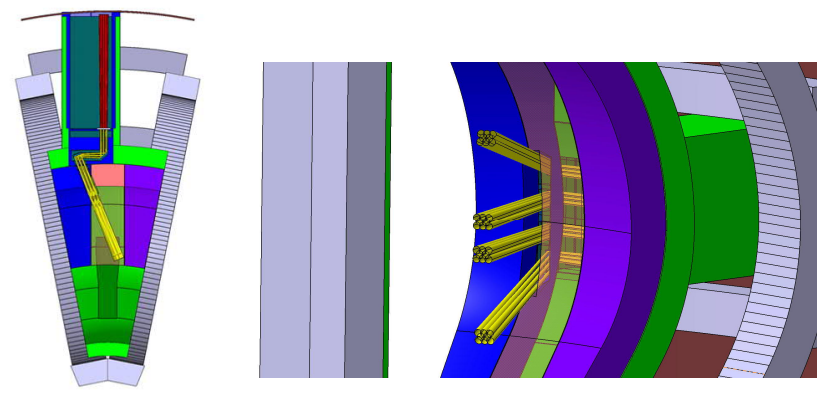

Fig. 7. Left: Top view and right: front view of DEMO MSA, yellow: indicates the EC waves, grey: TF coils, green: VV, blue, resp. violet / red: BB. The initial MSA port plug design has 4 drawers with 8 WGs (tbc.) with $63.5 \mathrm{~mm}$ diameter each.

\subsubsection{EC beam optics}

The focusing of the EC waves in the plasma is mandatory to be within a limited size in order to keep the NTM and ST power requirements as low as given in Table 1. Fig. 8 left shows the deposition profiles of a single $2 \mathrm{MW}$ beam. Assuming a seed island size of $3 \mathrm{~cm}$ and detection and reaction time of $0.5 \mathrm{~s}$ the island growth goes up to $6 \mathrm{~cm}$ and the required ECCD deposition half-width (1/e in power) is $\Delta \rho_{\mathrm{t}}=0.01$ (about $3 \mathrm{~cm}$ ), while the obtained deposition by GRAY [26] simulations was 0.0075 (about $2.25 \mathrm{~cm}$ ). There might be an influence on the beam broadening by scrape-off layer turbulences (e.g. blobs) [27] in front of the equatorial launching area, they shall be further studied and considered after they are quantified.

The configuration studies correspond to a beam waist radius (so far in vacuum) of $30 \mathrm{~mm}$ obtained with the focusing mid steering mirror located approximately $6500 \mathrm{~mm}$ from the absorption region in the plasma. The principle layout is shown in Fig 8 right, $\theta$ is the incidence angle, $\alpha$ is the poloidal and $\beta$ is the toroidal angle (definition cf. [28]). Considering that $\rho_{\mathrm{t}}=0.6$ $(\mathrm{q}=3 / 2)$ and $\rho_{\mathrm{t}}=0.77(\mathrm{q}=2)$ are reached with different injection angles $\alpha=16.8^{\circ}, \quad \beta=16.5^{\circ} \quad(q=2)$ and $\alpha=22.5^{\circ}, \beta=16.5^{\circ}(q=3 / 2)$ the value of $\theta$ will be around $53^{\circ}$.

\subsubsection{EC CAD design}




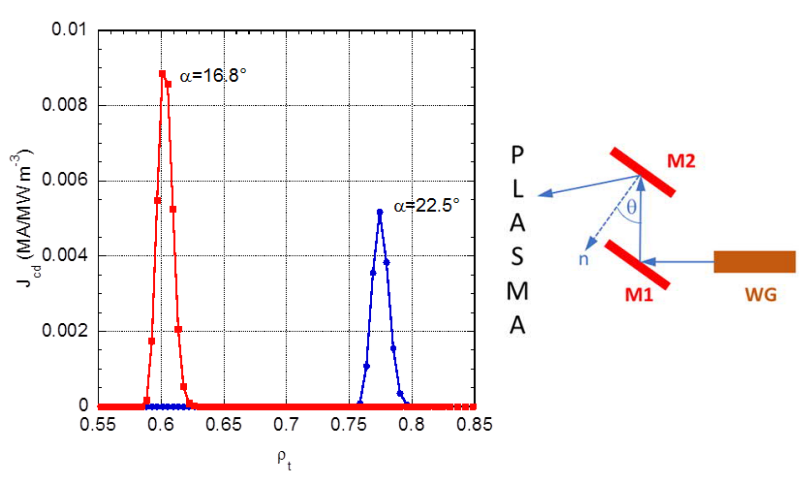

Fig. 8. Left: Current deposition profiles for $170 \mathrm{GHz}$ OM1 (ordinary mode) launched waves as result of GRAY code. Right: Top view of the DEMO mid steering antenna conceptual layout.

\subsubsection{EC neutronic loads}

Based on latests results of neutronic studies for two different design options of the RSA it was estimated that the maximum neutron induced damage for the preferred RSA option is $10 \mathrm{dpa}$ (displacements per atom) per 6 full power years in the front end components. These are not directly exposed to the plasma, but being recessed behind the BB and therefore mostly shielded. No showstopper was seen from the dpa studies. The selection of suitable material for the required dpa (such as stainless steel for the antennas or $\mathrm{CuCrZr}$ for the mirrors) considering its operational lifetime is necessary cf. [29][30]. Another issue is the nuclear heating. Here it needs more investigations and detailed cooling solutions, especially for small parts, which are not directly cooled, this issue could become critical. The neutronic analysis for the MSA is a next step after a design upgrade. For the OEWG it is expected to have a similar situation for the antennas (front ends) as for the RSA antenna openings done so far, therefore no dedicated study will be done.

\section{Conclusion}

Pre-conceptual HCD design integration studies were conducted. For NB, an updated beam duct reduced in opening size, by moving the focus point from the BB centre to the first wall, was proposed, to reduce neutron streaming, taking into account the fact that losing part of the beam halo power is unavoidable. However there were two issues outstanding, the TF coil winding pack heating and the transmission efficiency in the injector. For EC, two options for remote steering antenna (RSA) and one for mid steering antenna (MSA) were assessed in more detail, the OEWG concept is also considered but presently in a more generic way and not presented here. Analogous to the EC front steering solution of ITER, the idea for a DEMO EC MSA, where the movable mirrors are used - but in contrast to ITER - protected behind the $\mathrm{BB}$ has been introduced to the $\mathrm{HCD}$ project. It is presently the only option for DEMO with the possibility of having a large steering range and the required EC beam focusing for local ECCD to achieve MHD control. In the next steps, (i) the steering mirror size reduction, (ii) the launcher optimization in terms of remote maintainability, and (iii) abother option for NTM control, i.e. RSA with beam focussing by front mirrors, for a smaller steering range and individual suppression of each of the NTMs (at the $q=2$ and $q=3 / 2$ surfaces) by dedicated launchers, will be assessed.

\section{Acknowledgments}

This work has been carried out within the framework of the EUROfusion Consortium and has received funding from the EURATOM research and training programme 2014-2018 under grant agreement No 633053. The views and opinions expressed herein do not necessarily reflect those of the European Commission.

\section{References}

[1] T. Donné et al., J. Instrum. 12, 2017.

[2] G. Federici et al., Fus. Eng. Des. 136 (Part A), 2018, 729741.

[3] M. Siccinio et al., IAEA FEC 2018, Ahmedabad, India, 2018, Oct. FIP/P7-1, EFDA_D_2NRXR4

[4] M. Kovari, et al., Fusion Eng. Des. 89 (December (12)), 2014, 3054-69.

[5] P. Vincenzi et al., Fusion Eng. Des. 123, 2017, 473-476.

[6] G. Granucci et al., Nucl. Fusion 57, 2017, 8, 116009.

[7] Y.R. Martin et al., J. Phys. Conf. Ser. 123, 2008, 012033.

[8] T. P. Goodman et al., Phys. Rev. Lett. 106, 2011, 245002.

[9] H. Zohm et al., Nucl. Fusion 57, 2017, 086002.

[10] S. Garavaglia et al., Fus. Eng. Des. 136 (Part B), 2018, 1173-1177.

[11] T. Franke et al., IEEE Trans. Plasma Sci., 46 (5), 2018, 1633-1640.

[12] G. Grossetti et al., Nucl. Fusion 57, 2017, 116028.

[13] G. Grossetti et al., Fusion Eng. Des. 136 (Part A), 2018, 53-57.

[14] P. Sonato et al., New J. Phys. 18, 2016, 125002.

[15] P. Sonato et al., Nucl. Fusion 57, 2017, 056026.

[16] I. Fernández-Berceruelo et al., IEEE TPS 46 (7), 2018, 2708-2716.

[17] H.P.L. De-Esch, EFDA D 2MSR59, Nov2016.

[18] S. Zheng, A. Valentine, EFDA_D_2N7568, 2017.

[19] D. Pelowitz et al., MCNP $\overline{6}$ Users' Manual - Code Version 1.0, LA-CP-13-00634 Rev 0, May 2013.

[20] J. C. Sublet, J. W. G. Morgan, The FISPACT-II User Manual, CCFE-R(11)11 6 edition, 2014.

[21] C. Bachmann et al, Fusion Eng. Des. 136 (Part A), 2018, 87-95.

[22] U. Fischer et al., Fusion Eng. Des. 98-99, 2015, 21342137.

[23] Carr, M., et al., Rev. Sci. Instrum. 89 (8), 2018, 083506.

[24] P. Vincenzi et al., "Comparison of Neutral Beam Injection Options for EU DEMO Pulsed Scenario", P4.146 EPS, Belfast, 26-30 June 2017.

[25] P. Vincenzi et al., "Estimate of 3D wall heat loads due to Neutral Beam Injection in EU DEMO ramp-up phase", Nucl. Mat. Energy 18, 2019, 188-192.

[26] D. Farina, Fusion Sci. Technol. 52, 2007, 154-160.

[27] A. Snicker, et al., Nucl. Fusion 58, 2018, 016002.

[28] S. Garavaglia et al., EPJ Web of Conferences 147, 2017, 04002.

[29] A. Cufar, EFDA D 2MP8VW, 2017.

[30] A. Cufar et al., Equatorial electron cyclotron port plug neutronic analyses for the EU DEMO, Fusion Eng. Des., 2019 , in press. 